\title{
Conditional Knockout of Pdha1 in Mouse Hippocampus Impairs Cognitive Function: The Possible Involvement of Lactate
}

\section{OPEN ACCESS}

Edited by:

Xing Guo,

Nanjing Medical University, China

Reviewed by:

Yongye Huang,

Northeastern University, China

Yuanyuan Zhao,

Case Western Reserve University,

United States

Lan Zhang,

Capital Medical University, China

*Correspondence:

Libin Zhan

zlb/n@/nutcm.edu.cn

tThese authors have contributed equally to this work and share first

authorship

Specialty section:

This article was submitted to

Neurodegeneration,

a section of the journal

Frontiers in Neuroscience

Received: 31 August 2021

Accepted: 27 September 2021

Published: 14 October 2021

Citation:

Chen W, Sun X, Zhan L, Zhou W and Bi T (2021) Conditional Knockout of Pdha1 in Mouse Hippocampus Impairs Cognitive Function:

The Possible Involvement of Lactate.

Front. Neurosci. 15:767560

doi: 10.3389/fnins.2021.767560

\begin{abstract}
Wanxin Chen ${ }^{1+}$, Xiaoxia Sun ${ }^{1+}$, Libin Zhan ${ }^{2 *}$, Wen Zhou ${ }^{1}$ and Tingting Bi ${ }^{1}$
'School of Traditional Chinese Medicine \& School of Integrated Chinese and Western Medicine, Nanjing University of Chinese Medicine, Nanjing, China, ${ }^{2}$ Centre for Innovative Engineering Technology in Traditional Chinese Medicine, Liaoning University of Traditional Chinese Medicine, Shenyang, China
\end{abstract}

Background and Purpose: Neurodegenerative diseases are associated with metabolic disturbances. Pyruvate dehydrogenase E1 component subunit alpha (PDHA1) is an essential component in the process of glucose metabolism, and its deficiency exists in various diseases such as Alzheimer's disease (AD), epilepsy, Leigh's syndrome, and diabetes-associated cognitive decline. However, the exact role of PDHA1 deficiency in neurodegenerative diseases remains to be elucidated. In this study, we explored the effect of PDHA1 deficiency on cognitive function and its molecular mechanism.

Methods: A hippocampus-specific Pdha1 knockout $\left(\mathrm{Pdha1}^{-/-}\right)$mouse model was established, and behavioral tests were used to evaluate the cognitive function of mice. Transmission electron microscopy (TEM) was performed to observe the morphological changes of the hippocampus. The lactate level in the hippocampus was measured. Reverse transcription-quantitative polymerase chain reaction (RT-qPCR) and western blotting were used to explore the possible mechanism of the effect of PDHA1 on cognition.

Results: Pdha1 knockout damaged the spatial memory of mice and led to the ultrastructural disorder of hippocampal neurons. Lactate accumulation and abnormal lactate transport occurred in Pdha1-/- mice, and the cyclic AMP-protein kinase A-cAMP response element-binding protein (CAMP/PKA/CREB) pathway was inhibited.

Conclusion: Lactate accumulation caused by PDHA1 deficiency in the hippocampus may impair cognitive function by inhibiting the cAMP/PKA/CREB pathway.

Keywords: PDHA1, knockout mice, hippocampus, cognitive function, lactate

\section{INTRODUCTION}

Metabolic insufficiency occurs in neurodegenerative diseases, including Alzheimer's disease (AD; Turner, 2021). Pyruvate dehydrogenase complex (PDC) is a crucial enzyme in glucose metabolism, catalyzing the oxidative decarboxylation of pyruvate to acetyl-CoA, which links the cytoplasmic glycolytic pathway with the mitochondrial tricarboxylic acid cycle and oxidative phosphorylation 
(Patel et al., 2014). Pyruvate dehydrogenase E1 component subunit alpha (PDHA1) is a critical subunit of PDC. The clinical symptoms of PDC deficiency range from fatal lactic acidosis or progressive neuromuscular injury to chronic neurodegeneration (Robinson, 2006; Imbard et al., 2011; Patel et al., 2012; PavluPereira et al., 2021). These symptoms suggest that the nervous system is susceptible to perturbations in PDC activity due to its dependence on carbohydrate metabolism (Gray et al., 2014). PDHA1 deficiency has been found in a variety of neurodegenerative diseases, such as $\mathrm{AD}$, epilepsy, and Leigh's syndrome (Gavrilovici and Rho, 2020; Pawlosky et al., 2020; Gong et al., 2021). In addition, our previous study found that the expression of PDHA1 protein is decreased significantly in the hippocampi of rats with diabetes-associated cognitive decline (Shi et al., 2011).

Pyruvate dehydrogenase complex deficiency causes metabolic defects in the brain. On the one hand, the lack of PDC leads to insufficient energy supply to the brain, because the ATP needed by neurons is mainly produced in the mitochondria by oxidative phosphorylation of glucose via the tricarboxylic acid cycle (Bordone et al., 2019). On the other hand, PDC deficiency leads to the increase of lactate. In cells lacking PDC, pyruvate produced in the glycolytic pathway cannot enter the tricarboxylic acid cycle, however, it is converted to lactate, resulting in apparent aerobic glycolysis (Biswas et al., 1998). The role of lactate in the brain remains controversial. Lactate, produced from glucose through glycolysis, was initially believed to be a waste product; however, it is now considered an energy substrate and a signaling molecule (Magistretti and Allaman, 2018). Nevertheless, promoting lactate production by genetically manipulating metabolism can lead to oxidative stress and apoptosis in rat cortical neurons (HerreroMendez et al., 2009). One study found that lactate levels were elevated with age and correlated with more unsatisfactory memory performance in APP/PS1 mice (Harris et al., 2016). These findings suggest that changes in lactate metabolism may be a factor leading to the decline of cognitive function.

The hippocampus plays a vital role in learning and memory, and its structural and functional changes are associated with the development of neurodegenerative diseases related to cognitive decline (Bettio et al., 2017). Previous studies have not attracted enough attention regarding the effect of PDHA1 deficiency in the hippocampus on cognitive function. In order to solve this problem, we produced a novel hippocampus-specific Pdhal knockout $\left(\mathrm{Pdha1}^{-/-}\right)$mouse model. We hypothesized that lactate might be involved in cognitive impairment in $P d h a 1^{-/-}$ mice through the cyclic AMP-protein kinase A-cAMP response element-binding protein (cAMP/PKA/CREB) signaling pathway.

\section{MATERIALS AND METHODS}

\section{Animals}

Seven-month-old, half male and half female mice were used in all experiments. Pdha1 ${ }^{-/-}$mice were generated by Cyagen using the strategy outlined in Figure 1A. Eleven exons have been identified in Pdha1. Exon 4 was selected as conditional knockout region because deletion of exon 4 should result in the loss of function of the Pdhal gene. In the targeting vector, the Neo cassette was flanked by Rox sites, and cKO region was flanked by LoxP sites. The conditional KO allele was obtained after Dremediated recombination. The $\mathrm{KO}$ allele was obtained after Cremediated recombination. Figure 1B illustrates the genotyping strategy. For more information on genotyping, please see the Supplementary Material. The mice carrying KO allele were identified by genotyping, which were named $P d h a 1^{-/-}$mice and used in all experiments. Control wild-type mice were purchased from Vital River Laboratories (China). All mice were on a $\mathrm{C} 57 \mathrm{BL} / 6 \mathrm{~N}$ background. Mice were raised in the temperaturecontrolled $\left(24 \pm 2^{\circ} \mathrm{C}\right)$ specific pathogen-free animal experiment center of Nanjing University of Chinese Medicine, accepted a $12 \mathrm{~h}$ light/dark cycle with free access to food and water. All animal experiments were approved by the Animal Ethics Committee of Nanjing University of Chinese Medicine (Approval No. 201812A028).

\section{Behavioral Tests}

We used a series of behavioral tests, including the open field test (OFT), the novel object recognition (NOR) test, and the Morris water maze (MWM) test. The tests were performed on the following schedule: day 1, the OFT; day 2, the NOR test; and days 3-7, the MWM test.

\section{Open Field Test}

The OFT assessed spontaneous locomotor in mice. The test was performed in a $25 \mathrm{~cm} \times 25 \mathrm{~cm} \times 35 \mathrm{~cm}$ box. Mice were placed in the center of the box and allowed to explore freely for $5 \mathrm{~min}$. Behavioral patterns measured included total distance, velocity, time in the center $(5 \mathrm{~cm} \times 5 \mathrm{~cm})$, and entries in the center. The locomotion was recorded by video camera and registered on the computer. The square arena was cleaned with $75 \%$ alcohol after each trial.

\section{Novel Object Recognition Test}

The NOR test is used to evaluate short-term memory in mice (Pałucha-Poniewiera et al., 2021). First, a mouse was placed in a $25 \mathrm{~cm} \times 25 \mathrm{~cm} \times 35 \mathrm{~cm}$ box to adapt to $5 \mathrm{~min}$. Next, the mice were presented with two objects, $\mathrm{A}$ and $\mathrm{B}$, for $5 \mathrm{~min}$. One hour later, we replaced object $B$ with a novel object $C$ and repeated the trial. The square arena was cleaned with $75 \%$ alcohol after each trial. The interaction time with the novel object $\left(\mathrm{T}_{\mathrm{C}}\right)$ and familial object $\left(\mathrm{T}_{\mathrm{A}}\right)$ was recorded. The discrimination index is defined as $\mathrm{T}_{\mathrm{C}} /\left(\mathrm{T}_{\mathrm{A}}+\mathrm{T}_{\mathrm{C}}\right)$, which indicated new object recognition ability.

\section{Morris Water Maze Test}

The MWM test evaluated the spatial learning and memory performance of mice. The water maze consisted of a pool $(120 \mathrm{~cm}$ in diameter and filled with water maintained at $\left.24 \pm 1^{\circ} \mathrm{C}\right)$ and a platform ( $9 \mathrm{~cm}$ in diameter) submerged $1 \mathrm{~cm}$ under the water. On the first day, mice were put into the pool to swim freely for $60 \mathrm{~s}$ to adapt to the new condition without the platform before trials. During the 4-day training trial, mice were allowed to swim for $60 \mathrm{~s}$ to locate the hidden platform and rest for $60 \mathrm{~s}$ upon finding the platform. If the mouse failed to reach the platform within $60 \mathrm{~s}$, it was gently guided to the platform and allowed to stay for $60 \mathrm{~s}$. 
A

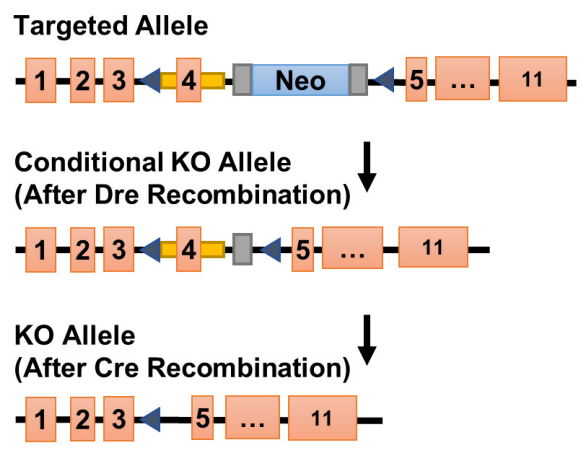

Exon $<$ LoxP site $\square$ Rox site $\square$ cKO region
B

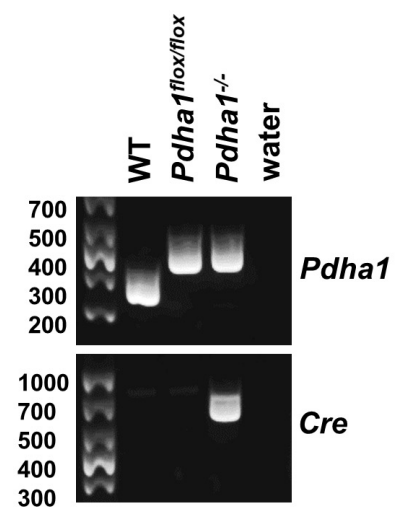

C

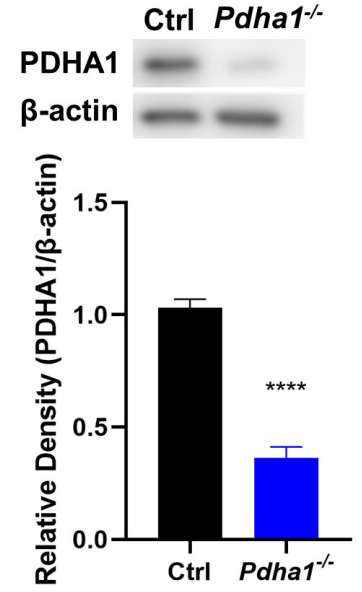

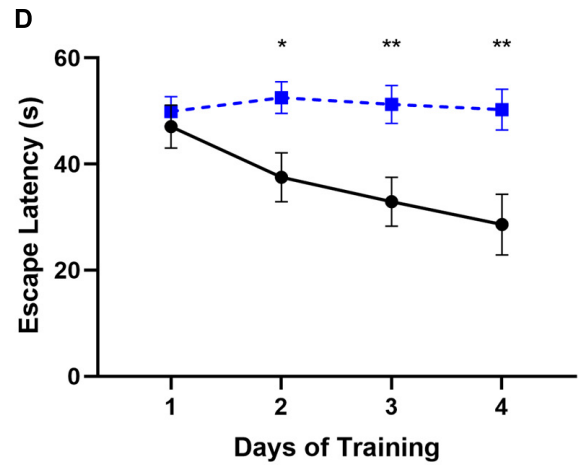

D

F

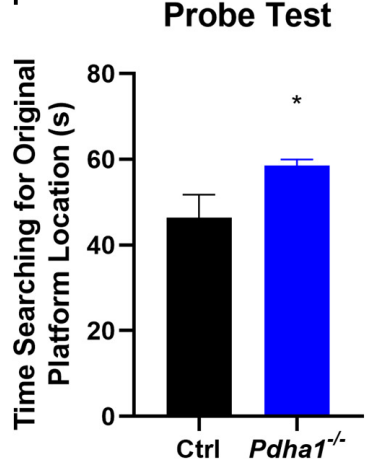

G

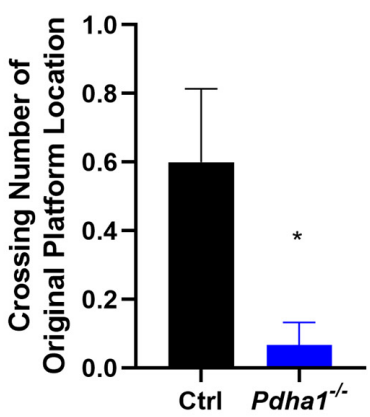

H

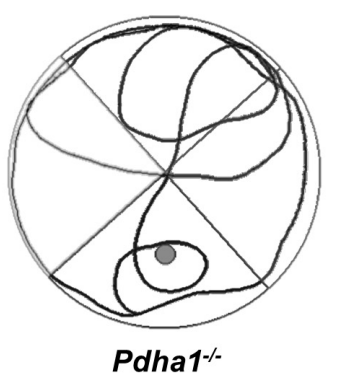

Pdha1--

E
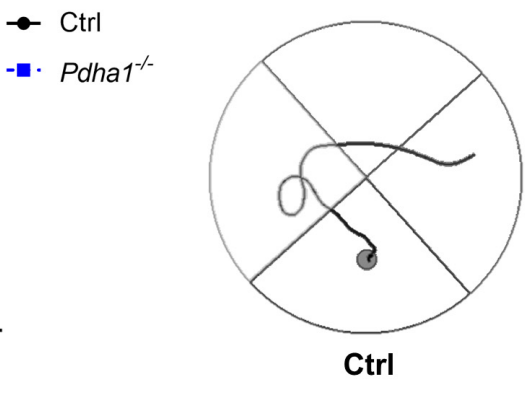

Probe Test

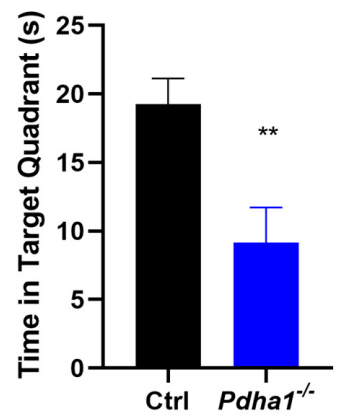

FIGURE 1| Pdha1-/- mice showed spatial memory impairment in the Morris water maze (MWM) test. (A) Schematic diagram of Pdha1 conditional knockout strategy. (B) Schematic diagram of PCR genotyping in mouse offspring. The floxed Pdha1 gene or wild-type gene were amplified as 394 and 281 bp, respectively. Cre was amplified as a $661 \mathrm{bp}$ fragment. (C) Representative immunoblots of PDHA1 protein and quantification of PDHA1 protein levels in the hippocampi of Pdha $1^{-/-}$mice and control mice $(n=4)$. (D) Escape latencies of the mice in the training trials $(n=15)$. (E) Representative swim track of Pdha $1^{-/-}$mice and control mice in the training trials. $(\mathbf{F}-\mathbf{H})$ The probe test, including time searching for the original platform location $\mathbf{( F )}$, number of crossings over the original platform location (G), and time in the target quadrant $(\mathbf{H})(n=15)$. All data are expressed as means \pm SEM, ${ }^{*} p<0.05,{ }^{* \star} p<0.01,{ }^{* \star \star *} p<0.0001$.

Mice were trained with four trials per day. The escape latency taken to reach the platform was measured automatically. Finally, the hidden platform was removed for the probe trial test. The time searching for the original platform, the number of platform crossings, and time spending in the target quadrant (the quadrant where the platform was located) were recorded. All data were measured by a camera and automated analysis system.

\section{Transmission Electron Microscopy}

Hippocampus samples were fixed with $2.5 \%$ glutaraldehyde. After rinsing with $0.1 \mathrm{M}$ phosphoric acid rinse solution, samples were placed in $1 \%$ osmium acid and fixed at $4^{\circ} \mathrm{C}$ for $2 \mathrm{~h}$. Samples were dehydrated in a graded series of ethanol, embedded, cured, and sliced into thin sections $(70 \mathrm{~nm})$ with an ultramicrotome (EM UC6, Leica, Germany). After staining with 3\% uranyl acetate 
and lead citrate, the samples were observed with the transmission electron microscope (JEM1230, JEOL, Japan).

\section{Lactate Measurement}

The lactate concentration of hippocampus samples was measured with Amplite ${ }^{\mathrm{TM}}$ Colorimetric L-Lactate Assay Kit (13815, AAT Bioquest, United States). The reaction was incubated at room temperature for $2 \mathrm{~h}$, protected from light, and the increase in absorbance ratio was detected at A575 nm/A605 nm. The data were analyzed according to the manufacturer's instructions.

\section{Western Blotting}

Hippocampus tissues were homogenized in RIPA buffer (P0012B, Beyotime, China) with a protease inhibitor cocktail (5871S, CST, United States) and phosphatase inhibitor cocktail (5870S, CST, United States). Extracted proteins were separated by $10 \%$ Sodium Dodecyl Sulfate-Polyacrylamide Gel Electrophoresis (SDS-PAGE) and transferred to PVDF membranes, and immunoblotted with the following antibodies: Pyruvate dehydrogenase E1 component subunit alpha (PDHA1) (sc377092, Santa Cruz, United States, 1:1000), Monocarboxylate transporter 1 (MCT1) (20139-1-AP, Proteintech, China 1:10000), Monocarboxylate transporter 2 (MCT2) (sc-166925, Santa Cruz,
United States,1:1000), Monocarboxylate transporter 4 (MCT4) (sc-376140, Santa Cruz, United States, 1:1000), phospho-Protein kinase A (PKA) (T197) (ab75991, Abcam, United Kingdom, 1:5000), PKA (sc-390548, Santa Cruz, United States, 1:1000), phospho-Cyclic AMP-responsive element-binding protein (CREB) (Ser133) (sc-81486, Santa Cruz, United States, 1:1000), CREB (sc-240, Santa Cruz, United States, 1:1000), HCAR1 (DF2766, Affinity, United States, 1:1000), and $\beta$-actin (3700S, CST, United States, 1:1000). The membranes were then treated with HRP-IgG antibody (BA-1054/BA1050, Boster, China, 1:2000). Antibody-bound protein bands were treated with a chemiluminescence solution (ECL, Tanon, China), and proteins were detected using an Amersham Imager 600 (General Electric Company, United States). Band intensities were analyzed with ImageQuant TL 1D software (GE Healthcare, United States).

\section{Reverse Transcription-Quantitative Polymerase Chain Reaction}

Total RNA of hippocampus samples was extracted using RNApure Tissue\&Cell Kit (CW0584S, Cwbio, China). The total RNA was converted to cDNA using PrimeScript RT Master Mix (Perfect Real Time) (RR036A, Takara, Japan) and the following reaction conditions: $37^{\circ} \mathrm{C}$ for $15 \mathrm{~min}, 85^{\circ} \mathrm{C}$ for
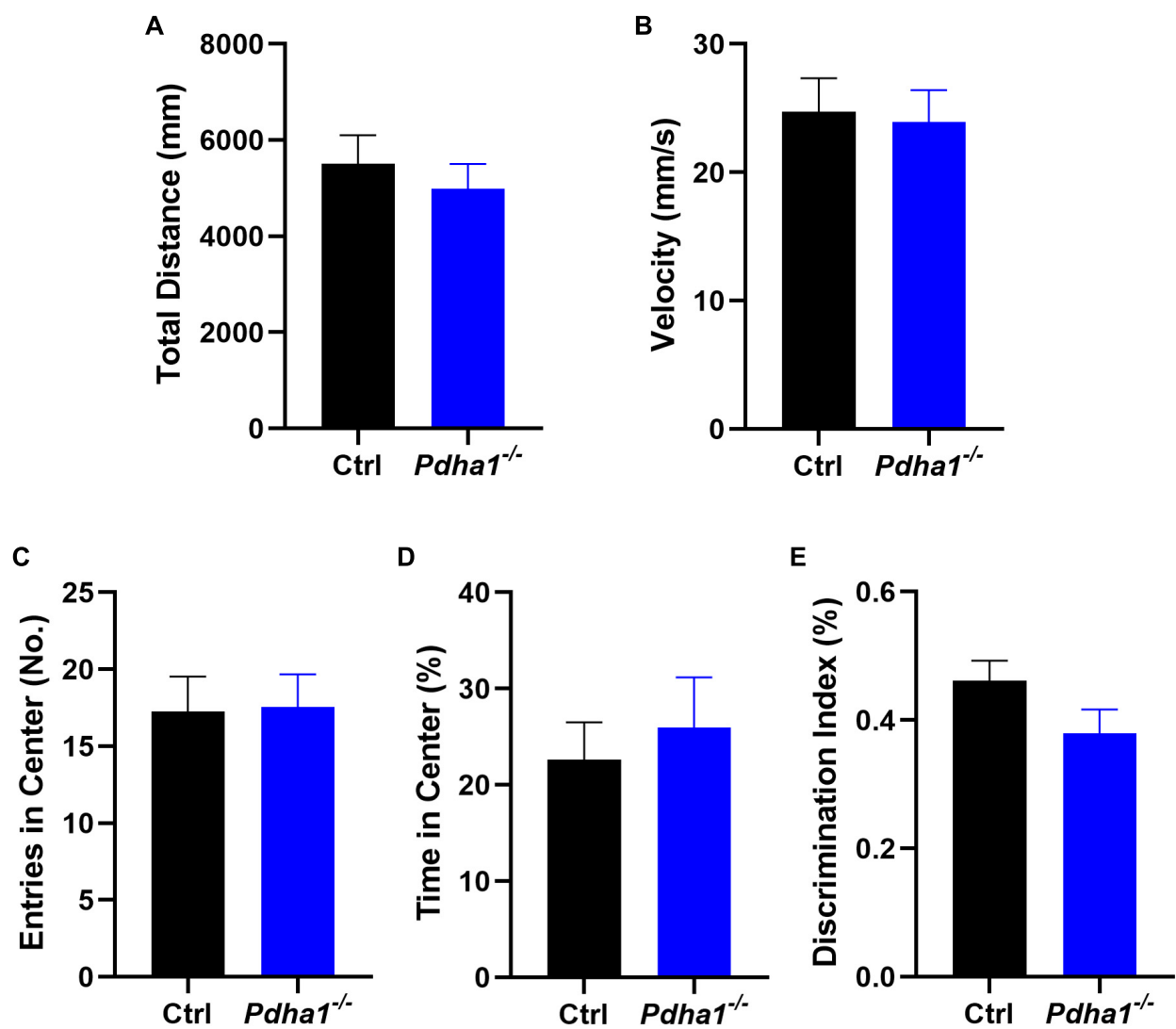

FIGURE $2 \mid P d h a 1^{-/-}$mice showed normal spontaneous locomotor activity and novel object recognition (NOR) memory. (A-D) Open field test (OFT), including total distance (A), velocity (B), entries in the center (C), and time in the center (D) $(n=15)$. (E) Discrimination index in the novel object recognition (NOR) test $(n=15)$. All data are expressed as means \pm SEM. 
$15 \mathrm{~s}$, and $4^{\circ} \mathrm{C}$ for 10 min. qPCR was conducted using SYBR Green Master Mix (Q141-02, Vazyme, China), and the reaction program used as follows: $95^{\circ} \mathrm{C}$ for $5 \mathrm{~min}, 95^{\circ} \mathrm{C}$ for $10 \mathrm{~s}$, and $60^{\circ} \mathrm{C}$ for $30 \mathrm{~s}$, and $95^{\circ} \mathrm{C}$ for $15 \mathrm{~s}, 60^{\circ} \mathrm{C}$ for $60 \mathrm{~s}$, and $95^{\circ} \mathrm{C}$ for $15 \mathrm{~s}$. Data were analyzed by the $\Delta \Delta \mathrm{Ct}$ method using $\beta$-actin as the reference gene. The primers were designed as follows: PKA, forward 5'-AGATCGTCCTGACCTTTGAGT$3^{\prime}$ and reverse 5'-GGCAAAACCGAAGTCTGTCAC-3'; CREB, forward 5'-AGCAGCTCATGCAACATCATC-3' and reverse $5^{\prime}$-AGTCCTTACAGGAAGACTGAACT-3'; $\beta$-actin, forward 5'-GGCTGTATTCCCCTCCATCG-3' and reverse 5'-CCAGTTGGTAACAATGCCATGT-3'.

\section{Statistical Analysis}

GraphPad Prism 8.3 statistical software (GraphPad Software, United States) was used for statistical analysis. All data are expressed as mean \pm standard error of mean (SEM). Statistical analyses were performed using Student's $t$-tests for comparing two groups. Training curves were analyzed by two-way repeated-measure ANOVAs and Sidak's multiple comparison tests. $p<0.05$ was considered statistically significant.

\section{RESULTS}

\section{Pdha1-/- Mice Show Spatial Memory Impairment}

To explore the effects of PDHA1 on cognitive function, we used the strategy shown in Figure 1 A to generate $P d h a 1^{-/-}$ mice. DNA prepared from tail snips of the knockout mice was analyzed by PCR, and the offspring of the dominant Pdhal gene (394 bp) and Cre gene (661 bp) were $P d h a 1^{-/-}$mice (Figure 1B). The hippocampi of mice were extracted to evaluate the expression of PDHA1. As expected, we observed that the expression of PDHA1 in the hippocampal protein extracts from Pdha1 ${ }^{-/}$mice decreased by $\sim 65 \%$ (Figure 1C). This result
A

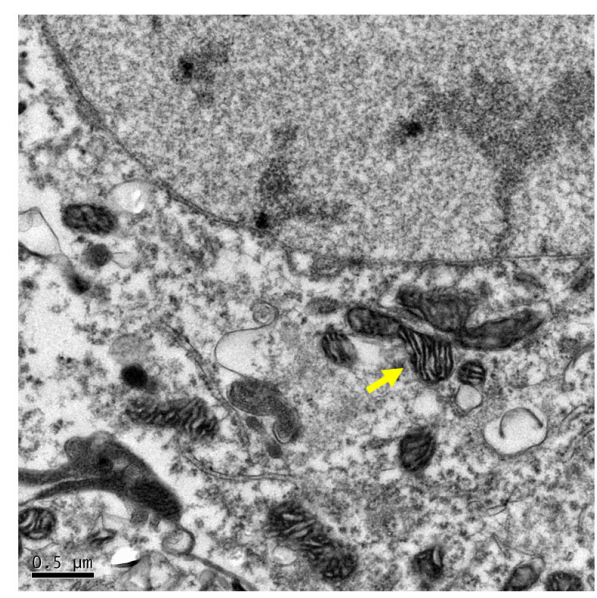

C

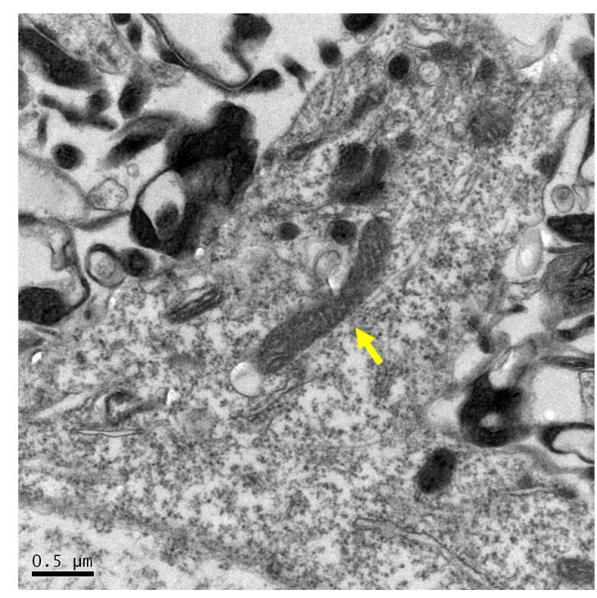

B

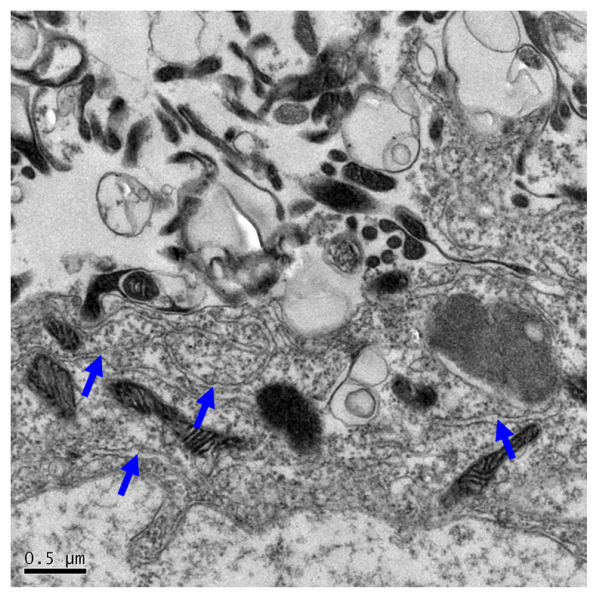

D

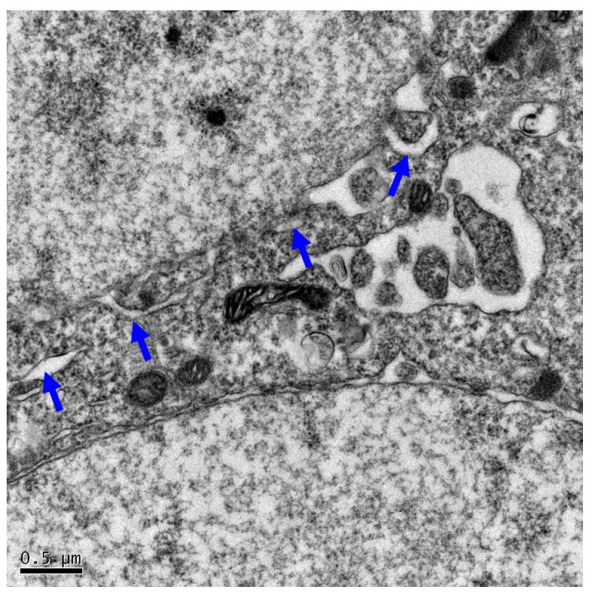

FIGURE 3 | Representative images of hippocampal neurons morphological changes evaluated by transmission electron microscopy (TEM). (A,B) The mitochondria and endoplasmic reticulum are structurally intact in control mice $(n=2)$. (C,D) By contrast, endoplasmic reticulum was dilatated and vesiculated, and mitochondria cristae appear vague in Pdha ${ }^{-1-}$ mice $(n=2)$. Yellow arrows indicate mitochondria, and blue arrows indicate endoplasmic reticulum. Scale bars $=0.5 \mu \mathrm{m}$. 
demonstrates that we successfully established a mouse model where the expression of PDHA1 is significantly reduced in the hippocampus.

Spatial memory was evaluated with the MWM test. In the training test, Pdha1 ${ }^{-/-}$mice typically took longer to reach the platform during the 4-day training period than the control mice (Figure 1D). At the same time, the trajectory map of $\mathrm{Pdha1}^{-/-}$mice was disorganized and purposeless (Figure 1E). In the space probe test, we found that $P d h a 1^{-/-}$ mice spent more time searching for the original platform location (Figure 1F). Compared with the control group, the crossing number of the original platform and the time spent in the target quadrant were significantly reduced in $P d h a 1^{-/-}$mice (Figures 1G,H). Overall, Pdha1 ${ }^{-/-}$mice showed spatial learning and memory impairment.

\section{Pdha1-/- Mice Do Not Show Obvious Spontaneous Locomotor Activity Changes or Novel Object Recognition Impairments}

To assess whether the behavioral changes of $\mathrm{Pdha1}^{-/-}$mice were associated with spontaneous locomotor impairments, the OFT was carried out (Figures 2A-D). Behavior parameters, including total distance (Figure 2A), velocity (Figure 2B), entries in the center (Figure 2C), and time in the center (\%) (Figure 2D), were not significantly different between $P d h a 1^{-/-}$mice and control mice, indicating that the impaired behavioral performance in Pdha $1^{-/-}$mice was not caused by reduced locomotor ability. In the NOR test, the recognition index of $\mathrm{Pdha1}^{-/-}$mice was slightly lower than that of control mice, but the difference was not significant (Figure 2E). Together, these results indicated normal spontaneous locomotor activity and novel object identification memory in Pdha1 ${ }^{-/-}$mice.

\section{Pdha1-/- Mice Display Abnormal Ultrastructure Morphology in Hippocampal Neurons}

We analyzed the organelle structure of the hippocampal neurons in mice by transmission electron microscopy (TEM). Healthy mitochondria and endoplasmic reticulum ultrastructure were observed in control mice (Figures 3A,B). However, in $\mathrm{Pdha1}^{-/-}$mice, the structure of the mitochondrial crest was disordered (Figure 3C), which is a typical feature of dysfunctional mitochondria. Moreover, the rough endoplasmic reticulum was dilated, vesiculated, and partially degranulated in hippocampal neurons of $\mathrm{Pdha1}^{-/-}$mice (Figure 3D). The results showed that the ultrastructure of hippocampal neurons in $P d h a 1^{-/-}$ mice was damaged.

\section{Pdha1-/- Mice Show Increased Lactate Levels and Abnormal Lactate Transport in the Hippocampus}

Lactic acidosis following glycolysis caused by decreased PDC activity is a key step related to metabolic reprogramming in the development of neurological disorders (Park et al., 2018).

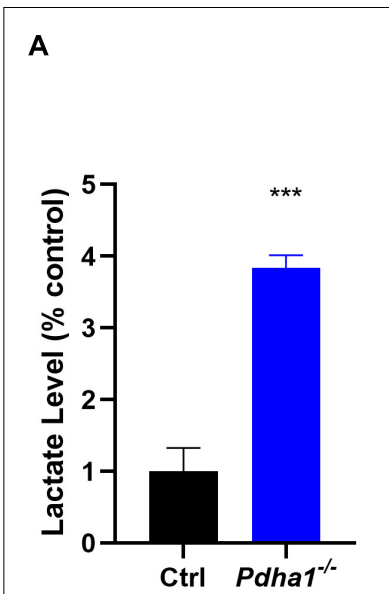

B

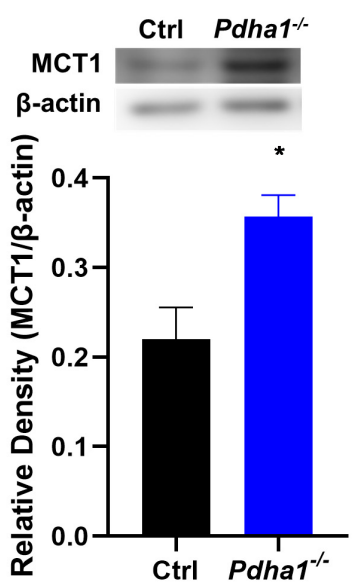

C

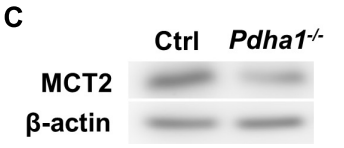

D

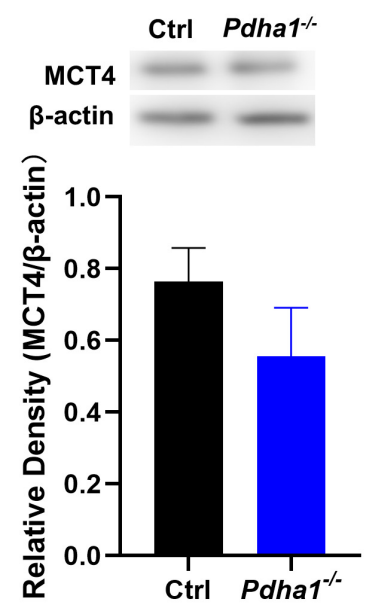

FIGURE 4 | $P$ dha1 ${ }^{-/}$- mice showed increased lactate levels and MCT expressions were changed in the hippocampus. (A) Quantification of lactate levels. (B) Representative immunoblots of MCT1 protein and quantification of MCT1 protein levels in the hippocampi of Pdha1-/- mice and control mice $(n=4)$. (C) Representative immunoblots of MCT2 protein and quantification of MCT2 protein levels in the hippocampi of $P d h a 1^{-1-}$ mice and control mice $(n=4)$. (D) Representative immunoblots of MCT4 protein and quantification of MCT4 protein levels in the hippocampi of $P d h a 1^{-1-}$ mice and control mice $(n=4)$. All data are expressed as means \pm SEM, ${ }^{\star} p<0.05,{ }^{\star \star \star} p<0.001$.

So, we first measured lactate levels in the hippocampus and found a significant increase in $\mathrm{Pdha1}^{-/-}$mice (Figure 4A). The transmembrane movement of lactate to or from cells is mainly mediated by monocarboxylate transporters (MCTs; Jha et al., 2016). In the brain, MCT1 is mainly expressed in endothelial cells and astrocytes. MCT2 mediates lactate uptake and is mainly expressed in neurons. MCT4 is expressed in astrocytes and plays a role in lactate output (Deitmer et al., 2019). Thus, we further examined the protein expression of MCTs. The results showed that, compared with the control group, the levels of MCT1 in Pdha1-/- mice increased significantly (Figure 4B). Conversely, we observed a significant decrease in MCT2 levels (Figure 4C). However, there were no significant differences in MCT4 levels between $P d h a 1^{-/}$mice and control mice (Figure 4D). These results suggested that the decline of 
cognitive function in $P d h a 1^{-/-}$mice may be related to abnormal lactate metabolism.

\section{PDHA1 Deficiency Inhibits the PKA/CREB Signaling Pathway}

The hippocampus is a critical tissue that regulates cognitive function, and the $\mathrm{CAMP} / \mathrm{PKA} / \mathrm{CREB}$ pathway plays an important role in brain function, so we analyzed the expression of PKA and CREB in the hippocampi of $\mathrm{Pdha1}^{-/-}$mice. The results showed significantly decreased PKA and CREB mRNA levels in $\mathrm{Pdha1}^{-/-}$mice (Figures 5A,B). Compared with the age-matched control group, the phosphorylation activities of PKA and CREB in $P d h a 1^{-/-}$mice were significantly inhibited (Figures $5 C, D$ ), indicating that PKA/CREB transduction was inhibited under the condition of PDHA1 deficiency. We speculated that the lactate accumulation could downregulate the cAMP/PKA/CREB signaling pathway because the lactate activates HCAR1, so we detected the protein expression of HCAR1 in the hippocampi of Pdha1 ${ }^{-/-}$mice. As we expected, the expression of HCAR1 in $\mathrm{Pdha1}^{-/-}$mice increased significantly (Figure 5E).

\section{DISCUSSION}

Although there is substantial evidence that PDHA1 deficiency is closely related to neurodegenerative diseases and cognitive impairment, it is unclear whether the loss of PDHA1 in the hippocampus will harm cognitive function. We used $P d h a 1^{-/-}$ mice to resolve this issue. Our current studies confirmed that PDHA1 deficiency in the hippocampus can lead to spatial memory impairment and ultrastructural damage to
A

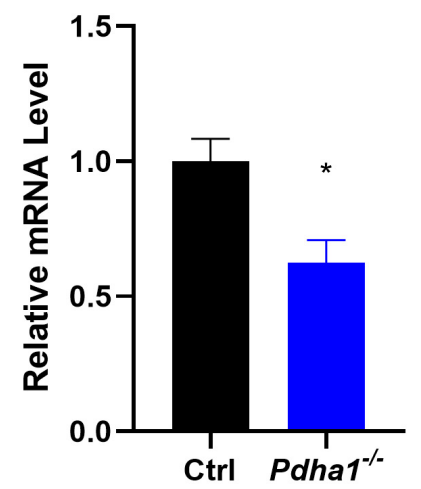

B

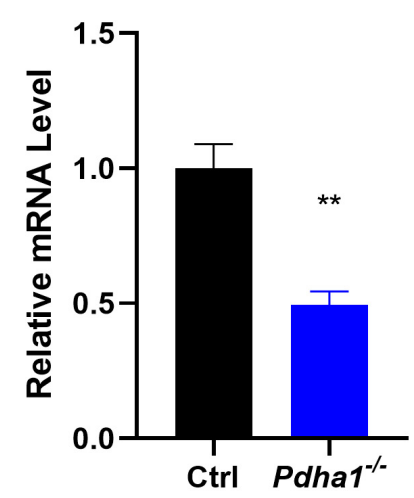

C

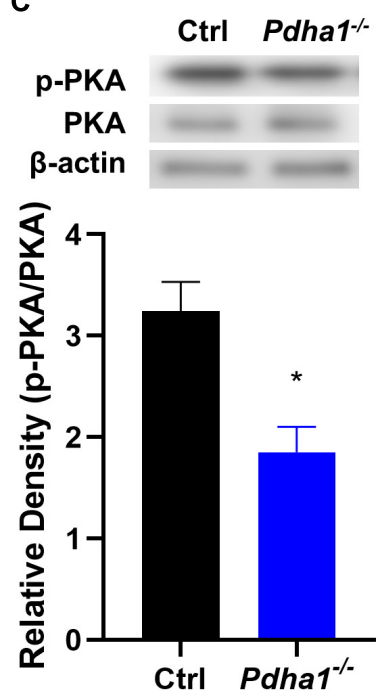

D

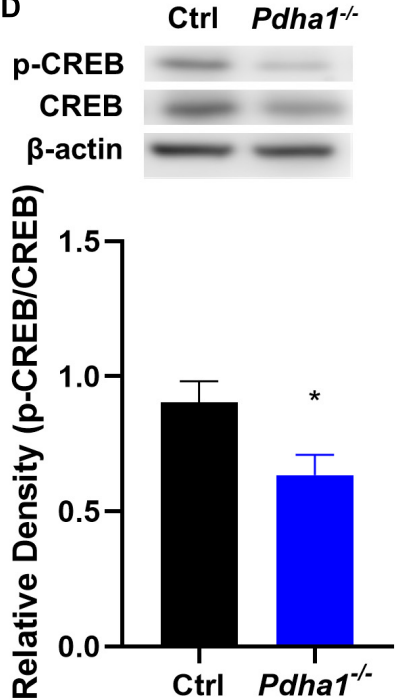

E

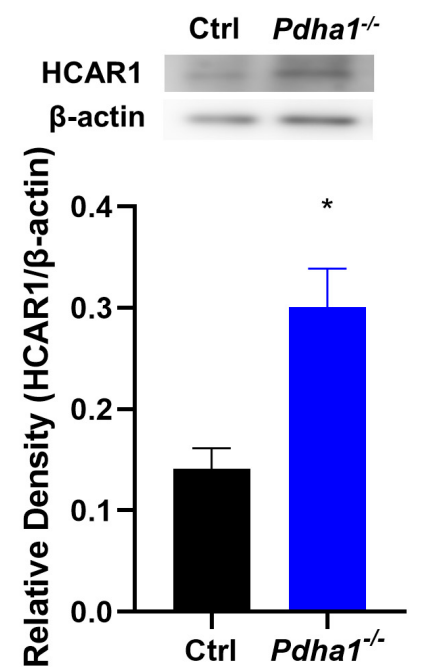

FIGURE 5 | Pdha1 ${ }^{-/-}$mice showed an inhibited PKA/CREB signaling pathway. (A,B) RT-qPCR determination of mRNA levels of PKA and CREB in hippocampi from Pdha $1^{-1-}$ mice and control mice $(n=4)$. (C) Representative immunoblots of $p$-PKA and PKA protein and quantification of p-PKA/PKA protein levels in the hippocampi of $P d h a 1^{-/-}$mice and control mice $(n=4)$. (D) Representative immunoblots of $p$-CREB and CREB protein, and quantification of $p$-CREB/CREB protein levels in the hippocampi of Pdha1-/- mice and control mice $(n=4)$. (E) Representative immunoblots of HCAR1 protein and quantification of HCAR1 protein levels in the hippocampi of Pdha1 ${ }^{-1-}$ mice and control mice $(n=4)$. Data are expressed as means \pm SEM, ${ }^{*} p<0.05,{ }^{*} p<0.01$. 


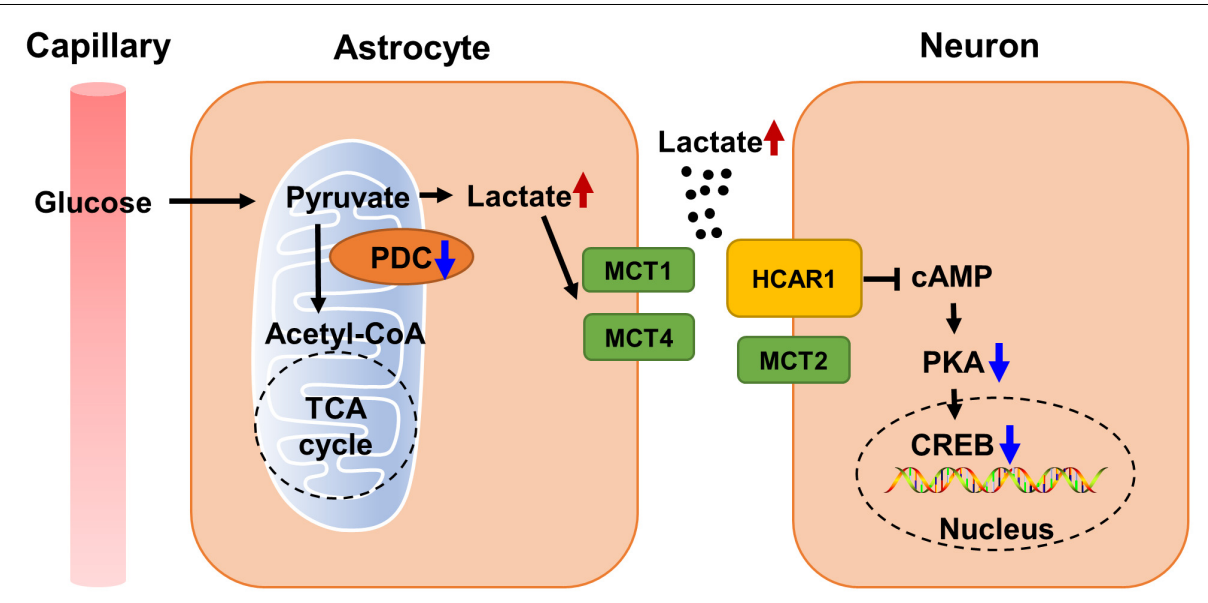

FIGURE 6 | Schematic diagram of cognitive function impaired by abnormal lactate metabolism in Pdha1 ${ }^{-/-}$mice. Due to decreased PDC activity, lactate abnormally increases, and excessive lactate transport through MCTs leads to tissue microenvironment acidification. Increased lactate also inhibits the cAMP/PKA/CREB signaling pathway by activating HCAR1, resulting in the decline of cognitive function in mice.

hippocampal neurons. In this case, the aerobic oxidation of glucose downstream of PDHA1 was blocked, which leads to the abnormal increase of the metabolite lactate. In addition, lactate transport was changed, which altered lactate homeostasis in the hippocampus. Elevated lactate may not only lead to tissue microenvironment acidification, but also act as a signaling molecule to inhibit the cAMP/PKA/CREB signaling pathway, thus hindering the formation of long-term memory (Figure 6). In conclusion, our study illustrates the important role of PDHA1 in learning and memory, and it may be a potential target in the treatment of cognitive decline.

In order to study whether hippocampal PDHA1 deficiency affects learning and memory, we conducted a series of behavioral tests on animals. The MWM test was used to evaluate the spatial learning and memory ability of mice. When animals cannot find the platform in the water maze, they cannot remember the spatial information they should have obtained during the training days, reflecting impairment of hippocampaldependent spatial cognition (Kim et al., 2016). Our data showed impaired spatial learning and memory abilities in $P d h a 1^{-/-}$ mice, suggesting that hippocampal PDHAl deficiency can lead to hippocampal dysfunction. It is well known that the hippocampus plays an essential role in storing and processing spatial information and transforming short-term memory into long-term memory. Interestingly, the short-term memory of the tested mice was not disturbed in the NOR test, indicating that the loss of PDHA1 in the hippocampus does not affect short-term memory. Furthermore, metabolic dysfunction caused by PDC deficiency can result in defective mitochondrial morphology (Perez-Siles et al., 2016; Park et al., 2018). We found that the deletion of Pdha1 led to the destruction of the ultrastructure of neurons in the hippocampus. To sum up, we concluded that the deletion of Pdhal affected spatial memory and the ultrastructure of neurons.

In order to further explain the possible mechanism of cognitive impairment caused by the loss of PDHA1, we measured the lactate levels in the hippocampi of mice. The results showed increased lactate levels in $P d h a 1^{-/-}$mice. Similar to our results, Pdha1 knockout tumor cells show an increase in extracellular acidification rate, an increase in extracellular lactate levels, and a decrease in ATP production, indicating that Pdha1-deleted cells cannot perform normal mitochondrial oxidative phosphorylation but are forced to undergo glycolysis (Zhong et al., 2017; Liu et al., 2019). The uptake of lactate by neurons is vital for establishing long-term memories (Camandola and Mattson, 2017). However, some researchers believe that excessive lactate impairs the function of neurons (Kweon and Suh, 2013; Scandella and Knobloch, 2019). The increase of lactate concentrations in the brain is usually related to brain diseases with cognitive impairments, such as leukoencephalopathy and AD (Scheper et al., 2007; Liguori et al., 2015; Cunnane et al., 2020). One study has shown that continuous lactate accumulation contributes to impaired adult neurogenesis, indicating that extracellular lactate levels should be strictly regulated (Wang et al., 2019). In summary, lactate may benefit the nervous system under physiological conditions, but the increase of pathological lactate may damage cognitive function.

There is evidence that the decrease of extracellular $\mathrm{pH}$ is accompanied by increased tissue lactate (Schneider et al., 1993). When lactate is produced, an essential way to acidify the extracellular environment is through MCTs (Spencer and Stanton, 2019). MCT1-MCT4 catalyze proton-coupled transport of lactate (Halestrap and Price, 1999). Brain cells rely on MCTs to maintain $\mathrm{pH}$ homeostasis, and the normal function of MCTs can prevent intracellular lactate accumulation and acidosis (Zhang et al., 2020). Our study found that the expression of MCT1 increased and MCT2 decreased in the hippocampi of Pdha1 ${ }^{-/-}$ mice, which may be an adaptation to the higher concentrations of lactate produced by glycolysis. The excess lactate produced in hippocampal cells of $P d h a 1^{-/-}$mice needs to be excreted by MCT1, which may lead to the compensatory increase of MCT1. MCT-mediated $\mathrm{H}^{+}$efflux exacerbates extracellular acidification 
(Ames et al., 2018). Normally, neurons absorb lactate through MCT2 as a source of energy (Kennedy and Dewhirst, 2010), whereas lactate utilization still requires the normal expression of PDHA1. The expression of MCT2 was downregulated in $\mathrm{Pdha1}^{-/-}$mice. One possible explanation is that neurons cannot utilize lactate as an energy substrate due to the loss of PDHA1. In summary, our results confirmed that lactate homeostasis in the hippocampi of $P d h a 1^{-/-}$mice was disrupted.

Lactate can be used as a direct agonist of hydroxycarboxylic acid receptor 1 (HCAR1, also known as GPR81) to participate in intercellular signal transduction (Lauritzen et al., 2014; Morland et al., 2017). The cAMP/PKA/CREB pathway is closely related to learning and memory. HCAR1 activation downregulates cAMP and weakens the signal transduction mediated by PKA (Langin, 2010). Activated PKA leads to phosphorylation of CREB, which initiates the transcription and translation of CREB target genes (Lonze and Ginty, 2002). CREB is a transcription factor that regulates neuronal growth, neuronal differentiation, neurogenesis, maturation of neurons, synaptic plasticity, spatial memory, and long-term memory (Sharma and Singh, 2020). Dysfunction of CREB is strongly correlated with several neurodegenerative diseases, including $\mathrm{AD}$, Parkinsonism, Huntington's disease, and ischemia (De Felice et al., 2007). Our study found that Pdhal knockout inhibited the cAMP/PKA/CREB signaling pathway, which may be mediated by lactate. Similar to our results, some studies have shown that lactate inhibits the HCAR1/PKA/CREB pathway in the brain of diabetes-associated cognitive decline rats (Dong et al., 2018; Zhao et al., 2018).

In conclusion, our results suggest that Pdhal knockout in the hippocampus leads to the accumulation of lactate and impairs gene expression behind learning and memory, resulting in memory and cognitive impairment. These results may help us understand the pathologies of memory or cognitive impairment, including neurodegenerative conditions such as $\mathrm{AD}$, diabetesassociated cognitive decline, and dementia.

This study has several limitations. First, we only studied the effect of PDHA1 deficiency on lactate, the final product of glycolysis, but the conversation of pyruvate to acetyl-CoA, glycolysis and TCA cycle remains to be further clarified. Second, we did not compare the degree of decline in expression of PDHA1 in different cell types in this mouse model. We hope to knock out Pdhal in different cell types in the future to

\section{REFERENCES}

Ames, S., Pastorekova, S., and Becker, H. M. (2018). The proteoglycan-like domain of carbonic anhydrase IX mediates non-catalytic facilitation of lactate transport in cancer cells. Oncotarget 9, 27940-27957. doi: 10.18632/oncotarg et. 25371

Bettio, L. E. B., Rajendran, L., and Gil-Mohapel, J. (2017). The effects of aging in the hippocampus and cognitive decline. Neurosci. Biobehav. Rev. 79, 66-86. doi: 10.1016/j.neubiorev.2017.04.030

Biswas, S., Ray, M., Misra, S., Dutta, D. P., and Ray, S. (1998). Is absence of pyruvate dehydrogenase complex in mitochondria a possible explanation of significant aerobic glycolysis by normal human leukocytes? FEBS Lett. 425, 411-414. doi: 10.1016/s0014-5793(98)00273-7 clarify the more detailed functions and mechanisms of PDHA1. Third, we only used 7-month-old mice for the study. Of note, neurodegenerative diseases are common chronic diseases that are associated with age (Hou et al., 2019). In the future, we will include mice of different months in the study to explore the relationship between cognitive impairment and age in Pdha1 ${ }^{-/-}$mice.

\section{DATA AVAILABILITY STATEMENT}

The original contributions presented in the study are included in the article/Supplementary Material, further inquiries can be directed to the corresponding author/s.

\section{ETHICS STATEMENT}

The animal study was reviewed and approved by the Animal Ethics Committee of Nanjing University of Chinese Medicine.

\section{AUTHOR CONTRIBUTIONS}

LZ conceived the idea, directed the project, designed the experiments, and involved in modifying the manuscript. WC and $\mathrm{XS}$ performed the experiments and analyzed the data. WC wrote the manuscript. LZ, XS, WZ, and TB edited the manuscript. All authors contributed to manuscript revision, read, and approved the submitted version.

\section{FUNDING}

This work was supported by the Key Project of the National Natural Science Foundation of China (81730111).

\section{SUPPLEMENTARY MATERIAL}

The Supplementary Material for this article can be found online at: https://www.frontiersin.org/articles/10.3389/fnins. 2021.767560/full\#supplementary-material

Bordone, M. P., Salman, M. M., Titus, H. E., Amini, E., Andersen, J. V., Chakraborti, B., et al. (2019). The energetic brain - A review from students to students. J. Neurochem. 151, 139-165. doi: 10.1111/jnc.14829

Camandola, S., and Mattson, M. P. (2017). Brain metabolism in health, aging, and neurodegeneration. EMBO J. 36, 1474-1492. doi: 10.15252/embj.201695810

Cunnane, S. C., Trushina, E., Morland, C., Prigione, A., Casadesus, G., Andrews, Z. B., et al. (2020). Brain energy rescue: an emerging therapeutic concept for neurodegenerative disorders of ageing. Nat. Rev. Drug Discov. 19, 609-633. doi: 10.1038/s41573-020-0072-x

De Felice, F. G., Wasilewska-Sampaio, A. P., Barbosa, A. C., Gomes, F. C., Klein, W. L., and Ferreira, S. T. (2007). Cyclic AMP enhancers and Abeta oligomerization blockers as potential therapeutic agents in Alzheimer's disease. Curr. Alzheimer Res. 4, 263-271. doi: 10.2174/156720507781077287 
Deitmer, J. W., Theparambil, S. M., Ruminot, I., Noor, S. I., and Becker, H. M. (2019). Energy dynamics in the brain: contributions of astrocytes to metabolism and $\mathrm{pH}$ homeostasis. Front. Neurosci. 13:1301. doi: 10.3389/fnins.2019. 01301

Dong, M., Ren, M., Li, C., Zhang, X., Yang, C., Zhao, L., et al. (2018). Analysis of metabolic alterations related to pathogenic process of diabetic encephalopathy rats. Front. Cell Neurosci. 12:527. doi: 10.3389/fncel.2018.00527

Gavrilovici, C., and Rho, J. M. (2020). Metabolic epilepsies amenable to ketogenic therapies: indications, contraindications, and underlying mechanisms. J. Inherit. Metab. Dis. 44, 42-53. doi: 10.1002/jimd.12283

Gong, K., Xie, L., Wu, Z. S., Xie, X., Zhang, X. X., and Chen, J. L. (2021). Clinical exome sequencing reveals a mutation in PDHA1 in Leigh syndrome: a case of a Chinese boy with lethal neuropathy. Mol. Genet. Genomic Med. 9:e1651. doi: $10.1002 / \mathrm{mgg} 3.1651$

Gray, L. R., Tompkins, S. C., and Taylor, E. B. (2014). Regulation of pyruvate metabolism and human disease. Cell Mol. Life Sci. 71, 2577-2604. doi: 10.1007/ s00018-013-1539-2

Halestrap, A. P., and Price, N. T. (1999). The proton-linked monocarboxylate transporter (MCT) family: structure, function and regulation. Biochem. J. 343(Pt 2), 281-299. doi: 10.1042/bj3430281

Harris, R. A., Tindale, L., Lone, A., Singh, O., Macauley, S. L., Stanley, M., et al. (2016). Aerobic glycolysis in the frontal cortex correlates with memory performance in wild-type mice but not the APP/PS1 mouse model of cerebral amyloidosis. J. Neurosci. 36, 1871-1878. doi: 10.1523/JNEUROSCI.3131-15. 2016

Herrero-Mendez, A., Almeida, A., Fernandez, E., Maestre, C., Moncada, S., and Bolanos, J. P. (2009). The bioenergetic and antioxidant status of neurons is controlled by continuous degradation of a key glycolytic enzyme by APC/CCdh1. Nat. Cell Biol. 11, 747-752. doi: 10.1038/ncb1881

Hou, Y., Dan, X., Babbar, M., Wei, Y., Hasselbalch, S. G., Croteau, D. L., et al. (2019). Ageing as a risk factor for neurodegenerative disease. Nat. Rev. Neurol. 15, 565-581. doi: 10.1038/s41582-019-0244-7

Imbard, A., Boutron, A., Vequaud, C., Zater, M., de Lonlay, P., de Baulny, H. O., et al. (2011). Molecular characterization of 82 patients with pyruvate dehydrogenase complex deficiency. Structural implications of novel amino acid substitutions in E1 protein. Mol. Genet. Metab. 104, 507-516. doi: 10.1016/j. ymgme.2011.08.008

Jha, M. K., Lee, I. K., and Suk, K. (2016). Metabolic reprogramming by the pyruvate dehydrogenase kinase-lactic acid axis: linking metabolism and diverse neuropathophysiologies. Neurosci. Biobehav. Rev. 68, 1-19. doi: 10.1016/j. neubiorev.2016.05.006

Kennedy, K. M., and Dewhirst, M. W. (2010). Tumor metabolism of lactate: the influence and therapeutic potential for MCT and CD147 regulation. Future Oncol. 6, 127-148. doi: 10.2217/fon.09.145

Kim, S., Kim, T., Lee, H. R., Jang, E. H., Ryu, H. H., Kang, M., et al. (2016). Impaired learning and memory in CD38 null mutant mice. Mol. Brain 9:16. doi: 10.1186/s13041-016-0195-5

Kweon, H. J., and Suh, B. C. (2013). Acid-sensing ion channels (ASICs): therapeutic targets for neurological diseases and their regulation. BMB Rep. 46, 295-304. doi: 10.5483/bmbrep.2013.46.6.121

Langin, D. (2010). Adipose tissue lipolysis revisited (again!): lactate involvement in insulin antilipolytic action. Cell Metab. 11, 242-243. doi: 10.1016/j.cmet.2010. 03.003

Lauritzen, K. H., Morland, C., Puchades, M., Holm-Hansen, S., Hagelin, E. M., Lauritzen, F., et al. (2014). Lactate receptor sites link neurotransmission, neurovascular coupling, and brain energy metabolism. Cereb. Cortex 24, 27842795. doi: $10.1093 /$ cercor/bht136

Liguori, C., Stefani, A., Sancesario, G., Sancesario, G. M., Marciani, M. G., and Pierantozzi, M. (2015). CSF lactate levels, tau proteins, cognitive decline: a dynamic relationship in Alzheimer's disease. J. Neurol. Neurosurg. Psychiatry 86, 655-659. doi: 10.1136/jnnp-2014-308577

Liu, L., Cao, J., Zhao, J., Li, X., Suo, Z., and Li, H. (2019). PDHAl gene knockout in human esophageal squamous cancer cells resulted in greater warburg effect and aggressive features in vitro and in vivo. Onco Targets Ther. 12, 9899-9913. doi: 10.2147/OTT.S226851

Lonze, B. E., and Ginty, D. D. (2002). Function and regulation of CREB family transcription factors in the nervous system. Neuron 35, 605-623. doi: 10.1016/ s0896-6273(02)00828-0
Magistretti, P. J., and Allaman, I. (2018). Lactate in the brain: from metabolic endproduct to signalling molecule. Nat. Rev. Neurosci. 19, 235-249. doi: 10.1038/ nrn.2018.19

Morland, C., Andersson, K. A., Haugen, O. P., Hadzic, A., Kleppa, L., Gille, A., et al. (2017). Exercise induces cerebral VEGF and angiogenesis via the lactate receptor HCAR1. Nat. Commun. 8:15557. doi: 10.1038/ncomms1 5557

Pałucha-Poniewiera, A., Podkowa, K., and Rafało-Ulińska, A. (2021). The group II mGlu receptor antagonist LY341495 induces a rapid antidepressant-like effect and enhances the effect of ketamine in the chronic unpredictable mild stress model of depression in C57BL/6J mice. Prog. Neuropsychopharmacol. Biol. Psychiatry 109:110239. doi: 10.1016/j.pnpbp.2020.110239

Park, S., Jeon, J. H., Min, B. K., Ha, C. M., Thoudam, T., Park, B. Y., et al. (2018). Role of the pyruvate dehydrogenase complex in metabolic remodeling: differential pyruvate dehydrogenase complex functions in metabolism. Diabetes Metab. J. 42, 270-281. doi: 10.4093/dmj.2018.0101

Patel, K. P., O’Brien, T. W., Subramony, S. H., Shuster, J., and Stacpoole, P. W. (2012). The spectrum of pyruvate dehydrogenase complex deficiency: clinical, biochemical and genetic features in 371 patients. Mol. Genet. Metab. 106, 385-394. doi: 10.1016/j.ymgme.2012.03.017

Patel, M. S., Nemeria, N. S., Furey, W., and Jordan, F. (2014). The pyruvate dehydrogenase complexes: structure-based function and regulation. J. Biol. Chem. 289, 16615-16623. doi: 10.1074/jbc.R114.563148

Pavlu-Pereira, H., Lousa, D., Tome, C. S., Florindo, C., Silva, M. J., de Almeida, I. T., et al. (2021). Structural and functional impact of clinically relevant E1alpha variants causing pyruvate dehydrogenase complex deficiency. Biochimie 183, 78-88. doi: 10.1016/j.biochi.2021.02.007

Pawlosky, R. J., Kashiwaya, Y., King, M. T., and Veech, R. L. (2020). A dietary ketone ester normalizes abnormal behavior in a mouse model of Alzheimer's Disease. Int. J. Mol. Sci. 21:1044. doi: 10.3390/ijms21031044

Perez-Siles, G., Ly, C., Grant, A., Drew, A. P., Yiu, E. M., Ryan, M. M., et al. (2016). Pathogenic mechanisms underlying X-linked Charcot-MarieTooth neuropathy (CMTX6) in patients with a pyruvate dehydrogenase kinase 3 mutation. Neurobiol. Dis. 94, 237-244. doi: 10.1016/j.nbd.2016. 07.001

Robinson, B. H. (2006). Lactic acidemia and mitochondrial disease. Mol. Genet. Metab. 89, 3-13. doi: 10.1016/j.ymgme.2006.05.015

Scandella, V., and Knobloch, M. (2019). Sensing the environment: extracellular lactate levels control adult neurogenesis. Cell Stem Cell 25, 729-731. doi: 10. 1016/j.stem.2019.11.008

Scheper, G. C., van der Klok, T., van Andel, R. J., van Berkel, C. G., Sissler, M., Smet, J., et al. (2007). Mitochondrial aspartyl-tRNA synthetase deficiency causes leukoencephalopathy with brain stem and spinal cord involvement and lactate elevation. Nat. Genet. 39, 534-539. doi: 10.1038/ng 2013

Schneider, U., Poole, R. C., Halestrap, A. P., and Grafe, P. (1993). Lactate-proton co-transport and its contribution to interstitial acidification during hypoxia in isolated rat spinal roots. Neuroscience 53, 1153-1162. doi: 10.1016/03064522(93)90497-4

Sharma, V. K., and Singh, T. G. (2020). CREB: a multifaceted target for Alzheimer's Disease. Curr. Alzheimer Res. 17, 1280-1293. doi: 10.2174/ 1567205018666210218152253

Shi, X., Lu, X. G., Zhan, L. B., Qi, X., Liang, L. N., Hu, S. Y., et al. (2011). The effects of the Chinese medicine $\mathrm{ZiBu}$ PiYin recipe on the hippocampus in a rat model of diabetes-associated cognitive decline: a proteomic analysis. Diabetologia 54, 1888-1899. doi: 10.1007/s00125-011-2147-z

Spencer, N. Y., and Stanton, R. C. (2019). The warburg effect, lactate, and nearly a century of trying to cure cancer. Semin. Nephrol. 39, 380-393. doi: 10.1016/j. semnephrol.2019.04.007

Turner, D. A. (2021). Contrasting metabolic insufficiency in aging and dementia. Aging Dis. 12, 1081-1096. doi: 10.14336/ad.2021.0104

Wang, J., Cui, Y., Yu, Z., Wang, W., Cheng, X., Ji, W., et al. (2019). Brain endothelial cells maintain lactate homeostasis and control adult hippocampal neurogenesis. Cell Stem Cell 25, 754.e9-767.e9. doi: 10.1016/j.stem.2019.09.009

Zhang, N., Guan, T., Shafiq, K., Xing, Y., Sun, B., Huang, Q., et al. (2020). Compromised lactate efflux renders vulnerability of oligodendrocyte precursor cells to metabolic stresses. ACS Chem. Neurosci. 11, 2717-2727. doi: 10.1021/ acschemneuro.0c00353 
Zhao, L., Dong, M., Ren, M., Li, C., Zheng, H., and Gao, H. (2018). Metabolomic analysis identifies lactate as an important pathogenic factor in diabetesassociated cognitive decline rats. Mol. Cell Proteomics 17, 2335-2346. doi: 10. 1074/mcp.RA118.000690

Zhong, Y., Li, X., Ji, Y., Li, X., Li, Y., Yu, D., et al. (2017). Pyruvate dehydrogenase expression is negatively associated with cell stemness and worse clinical outcome in prostate cancers. Oncotarget 8, 13344-13356. doi: 10.18632/ oncotarget.14527

Conflict of Interest: The authors declare that the research was conducted in the absence of any commercial or financial relationships that could be construed as a potential conflict of interest.
Publisher's Note: All claims expressed in this article are solely those of the authors and do not necessarily represent those of their affiliated organizations, or those of the publisher, the editors and the reviewers. Any product that may be evaluated in this article, or claim that may be made by its manufacturer, is not guaranteed or endorsed by the publisher.

Copyright (c) 2021 Chen, Sun, Zhan, Zhou and Bi. This is an open-access article distributed under the terms of the Creative Commons Attribution License (CC BY). The use, distribution or reproduction in other forums is permitted, provided the original author(s) and the copyright owner(s) are credited and that the original publication in this journal is cited, in accordance with accepted academic practice. No use, distribution or reproduction is permitted which does not comply with these terms. 